\title{
Effect of Compressibility on Hyperbolicity and Choke Flow Criterion of the Two-Phase Two-Fluid Model
}

Suneet Singh

Vincent A. Mousseau

September 2008

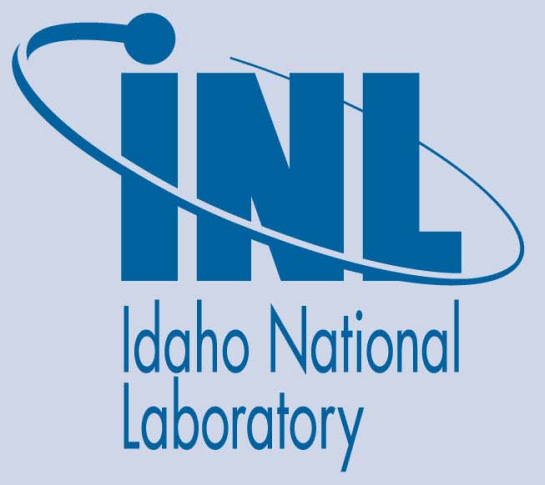

The INL is a U.S. Department of Energy National Laboratory operated by Battelle Energy Alliance 


\title{
Effect of Compressibility on Hyperbolicity and Choke Flow Criterion of the Two-Phase Two-Fluid Model
}

\author{
Suneet Singh \\ Vincent A. Mousseau
}

September 2008

\begin{abstract}
Idaho National Laboratory
Idaho Falls, Idaho 83415
\end{abstract}

http://www.inl.gov

Prepared for the

U.S. Department of Energy

Through the INL LDRD Program

Under DOE Idaho Operations Office

Contract DE-AC07-05ID14517 


\section{Table of Contents}

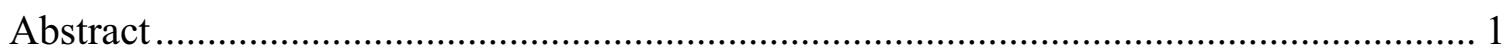

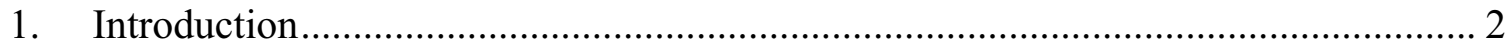

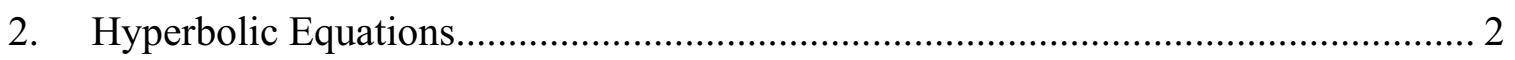

3. Characteristic equation for the two-phase two-fluid flow model ............................. 3

4. Review of eigenvalues of isothermal incompressible two-fluid model for two-phase

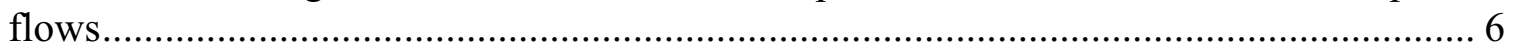

4.1. Correction term for the hyperbolicity …………......................................... 7

5. Compressibility effect on the characteristic equation ............................................. 9

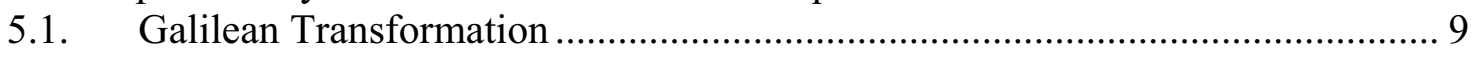

5.2. Eigenvalues for the zero slip velocity ……................................................ 10

5.3. Non-dimensional characteristic equation........................................................ 10

6. Numerical Solutions of the Benchmark problem.................................................. 13

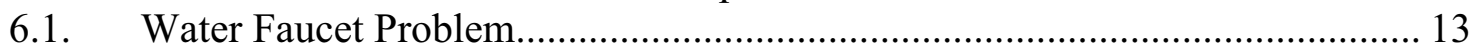

6.2. Numerical Results ................................................................................ 13

7. Series Solution of the characteristic equation for the small slip velocities............... 14

7.1. Analysis of the real roots of the characteristic equation .................................. 18

7.2. Complex roots of the characteristic equation................................................... 19

7.3. Choke Flow Criterion for the two phase flows ............................................... 19

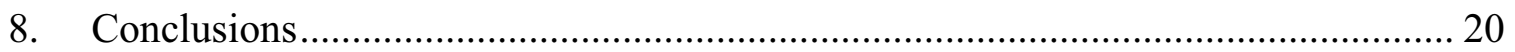

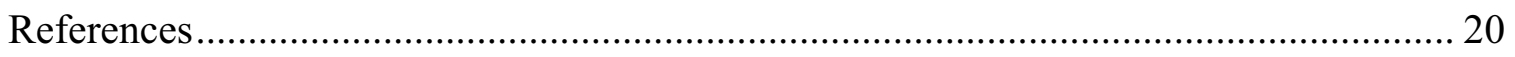




\begin{abstract}
The standard two-phase two-fluid model lacks hyperbolicity which results in oscillations in the numerical solutions. For the incompressible two-phase flows an exact correction term can be derived which when added to the momentum equations makes the model hyperbolic. No such straightforward approach exists for the similar compressible flows. In the current work, the effect of the compressibility on the characteristic equation is analyzed. It is shown that the hyperbolicity of the system depends only on the slip velocity and not on the phasic velocities, independently. Moreover, a slip Mach number $\left(M_{s}\right)$ is defined and a non-dimensional characteristic equation is derived. It is shown that for the small values of $M_{s}$, the effect of the compressibility on the hyperbolicity can be ignored. To verify the above analysis, the characteristic equation for the two-phase compressible flows is numerically solved and the result compared with the value obtained with the analytical solution for incompressible flows. Numerical solution of the twophase two-fluid model for the benchmark problem is used to further verify the abovementioned analysis. Furthermore, the eigenvalues of the characteristic equation are obtained as a power series expansion about the point $M_{s}=0$. These eigenvalues are used to develop a choking criterion for the compressible two-phase flows.
\end{abstract}




\section{Introduction}

The standard two-phase flow model employed for reactor safety [1] is not hyperbolic. It is quite common to rely on the numerical diffusion to deal with this problem [2]. However, this approach may not be satisfactory for higher order schemes. Moreover, even with the first order schemes, the numerical diffusion may not be sufficient to overcome the non-hyperbolicity for the operating regimes of the new reactor designs. Therefore, there is a need for the correction in the model in order to make it hyperbolic.

In the case of isothermal incompressible flows, growth factor due to non-hyperbolicity can be obtained analytically [3]. Moreover, for these flows, inclusion of a correction term in the two-fluid model results in a model which is hyperbolic. This term can be derived exactly for these flows [4]. For the compressible flows, one of the approaches is to use the abovementioned correction term (sometimes in conjunction with a virtual mass term) [5]. This model is known to fail for the case of compressible flows [6], therefore it is necessary to verify that this approach is satisfactory for the likely operating regimes for the existing as well as proposed nuclear reactor designs. In the current work, the model is analyzed, numerically as well as analytically, for some of the abovementioned operating regimes. In order to study the compressibility effect on the hyperbolicity a nondimensional characteristic equation is derived.

The characteristic equation for the compressible two-phase two-fluid model is a quartic equation. Although, an analytical solution for the quartic equation exists it is in the radical form and is not useful for the analysis. Therefore, a series solution for the nondimensional characteristic equation is obtained which is valid for the low slip velocities. The results obtained are compared with the results available in the literature [7]. Furthermore, a choking criterion for the compressible flows is obtained based on the obtained series solution.

\section{Hyperbolic Equations}

Consider a system of first order, quasilinear, partial differential equations given as,

$\mathbf{A} \frac{\partial \mathbf{F}}{\partial t}+\mathbf{B} \frac{\partial \mathbf{F}}{\partial x}=\mathbf{C}$

This system is considered to be hyperbolic if all the eigenvalues of the characteristic equation,

$|\mathbf{B}-\lambda \mathbf{A}|=0$

are real. The systems with complex eigenvalues are non-hyperbolic and results in the growing oscillations in the solutions. To avoid such oscillations system needs to be 
hyperbolic.

\section{Characteristic equation for the two-phase two-fluid flow model}

The governing equations for the two-phase two-fluid model are as follows,

Continuity Equation:

$\frac{\partial \alpha_{k} \rho_{k}}{\partial t}+\frac{\partial \alpha_{k} \rho_{k} u_{k}}{\partial x}=\Gamma_{k}$

Momentum equation (in the non-conservative form):

$\alpha_{k} \rho_{k} \frac{\partial u_{g}}{\partial t}+\alpha_{k} \rho_{k} u_{k} \frac{\partial u_{k}}{\partial x}+\alpha_{k} \frac{\partial P}{\partial x}=S_{k}$

Energy Equation (in the non-conservative form):

$\alpha_{k} \rho_{k} \frac{\partial U_{k}}{\partial t}+P \frac{\partial \alpha_{k}}{\partial t}+\alpha_{k} \rho_{k} u_{k} \frac{\partial U_{k}}{\partial x}+P \alpha_{k} \frac{\partial u_{k}}{\partial x}+P u_{k} \frac{\partial \alpha_{k}}{\partial x}=E_{k}$

In the above and subsequent equations $k$ is either $g$ (for the gas phase) or $l$ (for the liquid phase). The right hand side in the above equations does not have any derivatives and hence do not effect the hyperbolicity of the equations.

Using the product rule of the differentiation, continuity equation can be written as,

$\alpha_{k} \frac{\partial \rho_{k}}{\partial t}+\rho_{k} \frac{\partial \alpha_{k}}{\partial t}+\alpha_{k} u_{k} \frac{\partial \rho_{k}}{\partial x}+\rho_{k} u_{k} \frac{\partial \alpha_{k}}{\partial x}+\alpha_{k} \rho_{k} \frac{\partial u_{k}}{\partial x}=\Gamma_{k}$

From the equation of state, one can get, $\partial P / \partial \rho_{k}=c_{k}^{2}$. The continuity equation then becomes,

$\frac{\alpha_{k}}{c_{k}^{2}} \frac{\partial P}{\partial t}+\rho_{k} \frac{\partial \alpha_{k}}{\partial t}+\frac{\alpha_{k} u_{k}}{c_{k}^{2}} \frac{\partial P}{\partial x}+\rho_{k} u_{k} \frac{\partial \alpha_{k}}{\partial x}+\alpha_{k} \rho_{k} \frac{\partial u_{k}}{\partial x}=\Gamma_{k}$

Note that since,

$\alpha_{g}+\alpha_{l}=1$

therefore,

$\frac{\partial \alpha_{l}}{\partial t}=-\frac{\partial \alpha_{g}}{\partial t}$ 
and a similar relationship exists for the derivatives in space.

Comparing the system of the equations consisting of Eqs. (7), (4) and (5), with the matrix form of the equations [Eq. (1)], the matrices $\mathbf{A}, \mathbf{B}$ and vector $\mathbf{F}$ for the two-phase twofluid model can be written as,

$$
\begin{aligned}
\mathbf{A} & =\left[\begin{array}{cccccc}
\alpha_{g} / c_{g}^{2} & \rho_{g} & 0 & 0 & 0 & 0 \\
\alpha_{l} / c_{l}^{2} & -\rho_{l} & 0 & 0 & 0 & 0 \\
0 & 0 & \alpha_{g} \rho_{g} & 0 & 0 & 0 \\
0 & 0 & 0 & \alpha_{l} \rho_{l} & 0 & 0 \\
0 & P & 0 & 0 & \alpha_{g} \rho_{g} & 0 \\
0 & -P & 0 & 0 & 0 & \alpha_{l} \rho_{l}
\end{array}\right] \\
\mathbf{B} & =\left[\begin{array}{cccccc}
\alpha_{g} u_{g} / c_{g}^{2} & \rho_{g} u_{g} & \alpha_{g} \rho_{g} & 0 & 0 & 0 \\
\alpha_{l} u_{l} / c_{l}^{2} & -\rho_{l} u_{l} & 0 & \alpha_{l} \rho_{l} & 0 & 0 \\
\alpha_{g} & 0 & \alpha_{g} \rho_{g} u_{g} & 0 & 0 & 0 \\
\alpha_{l} & 0 & 0 & \alpha_{l} \rho_{l} u_{l} & 0 & 0 \\
0 & P u_{g} & P \alpha_{g} & 0 & \alpha_{g} \rho_{g} u_{g} & 0 \\
0 & -P u_{l} & 0 & P \alpha_{l} & 0 & \alpha_{l} \rho_{l} u_{l}
\end{array}\right]
\end{aligned}
$$

and

$$
\mathbf{F}=\left[\begin{array}{llllll}
p & \alpha_{g} & u_{g} & u_{l} & U_{g} & U_{l}
\end{array}\right]^{T}
$$

The vector, $\mathbf{C}$, consists of the source terms in the equation is irrelevant for the hyperbolicity of the model as discussed before.

The characteristic equation (see Eq.(2)) for the system of equations can be written as,

$$
\left|\begin{array}{cccccc}
\alpha_{g}\left(u_{g}-\lambda\right) / c_{g}^{2} & \rho_{g}\left(u_{g}-\lambda\right) & \alpha_{g} \rho_{g} & 0 & 0 & 0 \\
\alpha_{l}\left(u_{g}-\lambda\right) / c_{l}^{2} & -\rho_{l}\left(u_{l}-\lambda\right) & 0 & \alpha_{l} \rho_{l} & 0 & 0 \\
\alpha_{g} & 0 & \alpha_{g} \rho_{g}\left(u_{g}-\lambda\right) & 0 & 0 & 0 \\
\alpha_{l} & 0 & 0 & \alpha_{l} \rho_{l}\left(u_{l}-\lambda\right) & 0 & 0 \\
0 & P\left(u_{g}-\lambda\right) & P \alpha_{g} & 0 & \alpha_{g} \rho_{g}\left(u_{g}-\lambda\right) & 0 \\
0 & -P\left(u_{l}-\lambda\right) & 0 & P \alpha_{l} & 0 & \alpha_{l} \rho_{l}\left(u_{l}-\lambda\right)
\end{array}\right|=0(13)
$$

The expansion of the above determinant yields, 


$$
\left(u_{l}-\lambda\right)\left(u_{g}-\lambda\right)\left|\begin{array}{cccc}
\alpha_{g}\left(u_{g}-\lambda\right) / c_{g}^{2} & \rho_{g}\left(u_{g}-\lambda\right) & \alpha_{g} \rho_{g} & 0 \\
\alpha_{l}\left(u_{g}-\lambda\right) / c_{l}^{2} & -\rho_{l}\left(u_{l}-\lambda\right) & 0 & \alpha_{l} \rho_{l} \\
\alpha_{g} & 0 & \alpha_{g} \rho_{g}\left(u_{g}-\lambda\right) & 0 \\
\alpha_{l} & 0 & 0 & \alpha_{l} \rho_{l}\left(u_{l}-\lambda\right)
\end{array}\right|=0
$$

Two of the eigenvalues for the system are $u_{l}$ and $u_{g}$, which are real. Hence, these eigenvalues do not have any effect on the hyperbolicity of the system. Therefore, eigenvalues of the following equation will determine the hyperbolicity of the system,

$$
\left|\begin{array}{cccc}
\alpha_{g}\left(u_{g}-\lambda\right) / c_{g}^{2} & \rho_{g}\left(u_{g}-\lambda\right) & \alpha_{g} \rho_{g} & 0 \\
\alpha_{l}\left(u_{g}-\lambda\right) / c_{l}^{2} & -\rho_{l}\left(u_{l}-\lambda\right) & 0 & \alpha_{l} \rho_{l} \\
\alpha_{g} & 0 & \alpha_{g} \rho_{g}\left(u_{g}-\lambda\right) & 0 \\
\alpha_{l} & 0 & 0 & \alpha_{l} \rho_{l}\left(u_{l}-\lambda\right)
\end{array}\right|=0
$$

It should be noted that the above equation is the characteristic equation for the system with matrices $\mathbf{A}, \mathbf{B}$ and $\mathbf{F}$ as follows,

$$
\begin{aligned}
\mathbf{A} & =\left[\begin{array}{cccc}
\frac{\alpha_{g}}{c_{g}^{2}} & \rho_{g} & 0 & 0 \\
\frac{\alpha_{l}}{c_{l}^{2}} & -\rho_{l} & 0 & 0 \\
0 & 0 & \alpha_{g} \rho_{g} & 0 \\
0 & 0 & 0 & \alpha_{l} \rho_{l}
\end{array}\right] \\
\mathbf{B} & =\left[\begin{array}{cccc}
\frac{\alpha_{g} u_{g}}{c_{g}^{2}} & \rho_{g} u_{g} & \alpha_{g} \rho_{g} & 0 \\
\frac{\alpha_{l} u_{l}}{c_{l}^{2}} & -\rho_{l} u_{l} & 0 & \alpha_{l} \rho_{l} \\
\alpha_{g} & 0 & \alpha_{g} \rho_{g} u_{g} & 0 \\
\alpha_{l} & 0 & 0 & \alpha_{l} \rho_{l} u_{l}
\end{array}\right]
\end{aligned}
$$

and

$$
\mathbf{F}=\left[\begin{array}{llll}
p & \alpha_{g} & u_{g} & u_{l}
\end{array}\right]^{T}
$$

From the above matrices and Eqs. (4), (5) and (7), it can be inferred that this system represent the system of the equation for the isothermal two-phase flows (although source terms will be different for the isothermal case, they are irrelevant for the analysis of the hyperbolicity). Hence, it can be concluded that the analysis of the hyperbolicity for the 
isothermal system of the equations is also valid for the system of equations which include the energy equations.

\section{Review of eigenvalues of isothermal incompressible two-fluid model}

A review of the eigenvalues of the incompressible isothermal two-fluid two phase model is given in this section. The derivations and results given in this section have been obtained previously [3,4]. These results are reproduced here for the sake of completeness.

The characteristic equation of the incompressible case can be obtained by considering the case, $1 / c_{g}^{2}=1 / c_{l}^{2}=0$, Therefore, the characteristic equation can be written as (from Eq. $(15))$,

$$
\left|\begin{array}{cccc}
0 & \rho_{g}\left(u_{g}-\lambda\right) & \alpha_{g} \rho_{g} & 0 \\
0 & -\rho_{l}\left(u_{l}-\lambda\right) & 0 & \alpha_{l} \rho_{l} \\
\alpha_{g} & 0 & \alpha_{g} \rho_{g}\left(u_{g}-\lambda\right) & 0 \\
\alpha_{l} & 0 & 0 & \alpha_{l} \rho_{l}\left(u_{l}-\lambda\right)
\end{array}\right|=0
$$

Expanding on the last row, the above equation yields,

$$
\alpha_{l} \rho_{g}\left(u_{g}-\lambda\right)^{2}+\alpha_{g} \rho_{l}\left(u_{l}-\lambda\right)^{2}=0
$$

On further simplification, the above equation yields,

$$
\left(\alpha_{l} \rho_{g}+\alpha_{g} \rho_{l}\right) \lambda^{2}-2\left(u_{g} \alpha_{l} \rho_{g}+u_{l} \alpha_{g} \rho_{l}\right) \lambda+u_{g}^{2} \alpha_{l} \rho_{g}+u_{l}^{2} \alpha_{g} \rho_{l}=0
$$

The solution of the characteristic equation is as follows:

$$
\lambda=\frac{\left(u_{g} \alpha_{l} \rho_{g}+u_{l} \alpha_{g} \rho_{l}\right)}{\left(\alpha_{l} \rho_{g}+\alpha_{g} \rho_{l}\right)} \pm \frac{\sqrt{-\left(u_{g}-u_{l}\right)^{2} \alpha_{g} \rho_{l} \alpha_{l} \rho_{g}}}{\left(\alpha_{l} \rho_{g}+\alpha_{g} \rho_{l}\right)}
$$

It may be noted from the above equation that the eigenvalues are complex except when either $u_{g}=u_{l}$ or the system approaches single phase $\left(\alpha_{g} \rightarrow 0\right.$ or $\left.\alpha_{l} \rightarrow 0\right)$. One more case which should be considered is the case when $\partial \alpha_{g} / \partial x=0$. In this case, the continuity equation becomes,

$$
\frac{\alpha_{k}}{c_{k}^{2}} \frac{\partial P}{\partial t}+\rho_{k} \frac{\partial \alpha_{k}}{\partial t}+\frac{\alpha_{k} u_{k}}{c_{k}^{2}} \frac{\partial P}{\partial x}+\alpha_{k} \rho_{k} \frac{\partial u_{k}}{\partial x}=\Gamma_{k}
$$


the corresponding matrix $\mathbf{B}$ (for incompressible flow i..e. with $1 / c_{g}^{2}=1 / c_{l}^{2}=0$ ) modifies to,

$$
\mathbf{B}=\left[\begin{array}{cccc}
0 & 0 & \alpha_{g} \rho_{g} & 0 \\
0 & 0 & 0 & \alpha_{l} \rho_{l} \\
\alpha_{g} & 0 & \alpha_{g} \rho_{g} u_{g} & 0 \\
\alpha_{l} & 0 & 0 & \alpha_{l} \rho_{l} u_{l}
\end{array}\right]
$$

The matrix $\mathbf{A}$, however, is not changed.

In view of the modification in matrix $\mathbf{B}$, the characteristic equation becomes.

$$
\left|\begin{array}{cccc}
0 & -\lambda & \alpha_{g} \rho_{g} & 0 \\
0 & -\lambda & 0 & \alpha_{l} \rho_{l} \\
\alpha_{g} & 0 & \alpha_{g} \rho_{g}\left(u_{g}-\lambda\right) & 0 \\
\alpha_{l} & 0 & 0 & \alpha_{l} \rho_{l}\left(u_{l}-\lambda\right)
\end{array}\right|=0
$$

The above equation on expansion of the determinant yields,

$$
\alpha_{l} \rho_{g} \lambda\left(u_{g}-\lambda\right)+\alpha_{g} \rho_{l} \lambda\left(u_{l}-\lambda\right)=0
$$

The roots of the above equation are,

$$
\lambda=0, \frac{\left(u_{g} \alpha_{l} \rho_{g}+u_{l} \alpha_{g} \rho_{l}\right)}{\left(\alpha_{l} \rho_{g}+\alpha_{g} \rho_{l}\right)}
$$

which are real.

Since, the eigenvalues are complex except for the specific cases discussed above $\left(u_{g}=u_{l}, \alpha_{g} \rightarrow 0, \alpha_{l} \rightarrow 0\right.$ and $\left.\partial \alpha_{g} / \partial x=0\right)$, hence in general system of the equations for the incompressible two-phase flow is non-hyperbolic.

\subsection{Correction term for the hyperbolicity}

In presence of the correction term $\left(p_{i} \partial \alpha_{k} / \partial x\right)$ the momentum equation becomes,

$$
\alpha_{k} \rho_{k} \frac{\partial u_{g}}{\partial t}+\alpha_{k} \rho_{k} u_{k} \frac{\partial u_{k}}{\partial x}+\alpha_{k} \frac{\partial P}{\partial x}+p_{i} \frac{\partial \alpha_{k}}{\partial x}=S_{k}
$$


The matrix $\mathbf{B}$ is modified to the following,

$$
\mathbf{B}=\left[\begin{array}{cccc}
0 & \rho_{g} u_{g} & \alpha_{g} \rho_{g} & 0 \\
0 & -\rho_{l} u_{l} & 0 & \alpha_{l} \rho_{l} \\
\alpha_{g} & p_{i} & \alpha_{g} \rho_{g} u_{g} & 0 \\
\alpha_{l} & -p_{i} & 0 & \alpha_{l} \rho_{l} u_{l}
\end{array}\right]
$$

There is no change in the matrix A. The characteristic equation then can be given as,

$$
\left|\begin{array}{cccc}
0 & \rho_{g}\left(u_{g}-\lambda\right) & \alpha_{g} \rho_{g} & 0 \\
0 & -\rho_{l}\left(u_{l}-\lambda\right) & 0 & \alpha_{l} \rho_{l} \\
\alpha_{g} & p_{i} & \alpha_{g} \rho_{g}\left(u_{g}-\lambda\right) & 0 \\
\alpha_{l} & -p_{i} & 0 & \alpha_{l} \rho_{l}\left(u_{l}-\lambda\right)
\end{array}\right|=0
$$

The above equation can be expanded to,

$\left(\alpha_{l} \rho_{g}+\alpha_{g} \rho_{l}\right) \lambda^{2}-2\left(u_{g} \alpha_{l} \rho_{g}+u_{l} \alpha_{g} \rho_{l}\right) \lambda+u_{g}^{2} \alpha_{l} \rho_{g}+u_{l}^{2} \alpha_{g} \rho_{l}-p_{i}=0$

and the corresponding roots become,

$$
\lambda=\frac{\left(u_{g} \alpha_{l} \rho_{g}+u_{l} \alpha_{g} \rho_{l}\right)}{\left(\alpha_{l} \rho_{g}+\alpha_{g} \rho_{l}\right)} \pm \frac{\sqrt{-\left(u_{g}-u_{l}\right)^{2} \alpha_{g} \rho_{l} \alpha_{l} \rho_{g}+p_{i}\left(\alpha_{l} \rho_{g}+\alpha_{g} \rho_{l}\right)}}{\left(\alpha_{l} \rho_{g}+\alpha_{g} \rho_{l}\right)}
$$

It is obvious from the above equation, that choosing

$$
p_{i}=\frac{\left(u_{g}-u_{l}\right)^{2} \alpha_{g} \rho_{l} \alpha_{l} \rho_{g}}{\alpha_{g} \rho_{l}+\alpha_{l} \rho_{g}}
$$

will make the system of the equations hyperbolic. In fact, system is hyperbolic for any value larger than the above mentioned value of the correction term. However, minimum value of this term is used in order to have minimum possible change in the original model. It is also important to note that the choice of the minimum required value will keep the real part of the eigenvalues exactly same as the original model while eliminating the imaginary part.

Note that the correction term goes to zero for all the cases in which the original model itself is hyperbolic $\left(u_{g}=u_{l}, \alpha_{g} \rightarrow 0, \alpha_{l} \rightarrow 0\right.$ and $\left.\partial \alpha_{g} / \partial x=0\right)$. 


\section{Compressibility effect on the characteristic equation}

For the compressible two-phase flows the characteristic equation is a quartic equation and hence the eigenvalues can not be obtained analytically in the simple form. Characteristic equation (Eq. (15)) is expanded and separated into incompressible and compressible parts as follows,

$$
\left[-\frac{\alpha_{l} \rho_{g}}{c_{l}^{2}}\left(u_{g}-\lambda\right)^{2}\left(u_{l}-\lambda\right)^{2}-\frac{\alpha_{g} \rho_{l}}{c_{g}^{2}}\left(u_{g}-\lambda\right)^{2}\left(u_{l}-\lambda\right)^{2}\right]+\left[\alpha_{l} \rho_{g}\left(u_{g}-\lambda\right)^{2}+\alpha_{g} \rho_{l}\left(u_{l}-\lambda\right)^{2}\right]=0
$$

In the above equation these two parts are given in two separate square brackets. It should be noted that the first square bracket of the above characteristic equation is zero for the incompressible flow. The two square brackets in the subsequent equations also have the compressible and incompressible parts, respectively.

The above equation can be written as follows,

$$
-\frac{\left(c_{g}^{2} \alpha_{l} \rho_{g}+c_{l}^{2} \alpha_{g} \rho_{l}\right)}{c_{g}^{2} c_{l}^{2}}\left[\left(u_{g}-\lambda\right)^{2}\left(u_{l}-\lambda\right)^{2}\right]+\left[\alpha_{l} \rho_{g}\left(u_{g}-\lambda\right)^{2}+\alpha_{g} \rho_{l}\left(u_{l}-\lambda\right)^{2}\right]=0
$$

\subsection{Galilean Transformation}

Substituting,

$\lambda=Y+\frac{u_{g}+u_{l}}{2}$

one gets,

$-\frac{\left(c_{g}^{2} \alpha_{l} \rho_{g}+c_{l}^{2} \alpha_{g} \rho_{l}\right)}{c_{g}^{2} c_{l}^{2}}\left[(\Delta u-Y)^{2}(\Delta u+Y)^{2}\right]+\left[\alpha_{l} \rho_{g}(\Delta u-Y)^{2}+\alpha_{g} \rho_{l}(\Delta u+Y)^{2}\right]=0$

where,

$\Delta u=\left(u_{g}-u_{l}\right) / 2$

The above substitution is equivalent to a Galilean transformation such that after the transformation one phase moves with the velocity $\Delta u$ and the other phase with velocity $-\Delta u$. It needs to be emphasized that the imaginary part of the solution of Eq. (37) will be same as that of the original characteristic equation. Therefore, the analysis of the hyperbolicity of the system can be carried out using Eq. (37) instead of using Eq (35). Since, Eq. (37) is written only in the terms of the slip velocity $\Delta u$, therefore, the 
hyperbolicity of the system depends only on the slip velocity and not on the phasic velocities $u_{g}$ and $u_{l}$, independently.

Dividing Eq. (37) by $\alpha_{l} \rho_{g}+\alpha_{g} \rho_{l}$, one gets,

$-\frac{\left(c_{g}^{2} \alpha_{l} \rho_{g}+c_{l}^{2} \alpha_{g} \rho_{l}\right)}{c_{g}^{2} c_{l}^{2}\left(\alpha_{l} \rho_{g}+\alpha_{g} \rho_{l}\right)}\left[\left(\Delta u^{2}-Y^{2}\right)^{2}\right]+\left[\frac{\alpha_{l} \rho_{g}(\Delta u-Y)^{2}+\alpha_{g} \rho_{l}(\Delta u+Y)^{2}}{\left(\alpha_{l} \rho_{g}+\alpha_{g} \rho_{l}\right)}\right]=0$.

Defining a sound speed, $c_{m 0}^{2}=\frac{c_{g}^{2} c_{l}^{2}\left(\alpha_{l} \rho_{g}+\alpha_{g} \rho_{l}\right)}{\left(c_{g}^{2} \alpha_{l} \rho_{g}+c_{l}^{2} \alpha_{g} \rho_{l}\right)}$, the above equation can be written as,

$-\frac{1}{c_{m 0}^{2}}\left[\left(\Delta u^{2}-Y^{2}\right)^{2}\right]+\frac{1}{\left(\alpha_{l} \rho_{g}+\alpha_{g} \rho_{l}\right)}\left[\alpha_{l} \rho_{g}(\Delta u-Y)^{2}+\alpha_{g} \rho_{l}(\Delta u+Y)^{2}\right]=0$

\subsection{Eigenvalues for the zero slip velocity}

In case $\Delta u=0$ (i.e. $\left.u_{g}=u_{l}\right)$ the above equation becomes,

$-\frac{Y^{4}}{c_{m 0}^{2}}+Y^{2}=0$

The roots of the above equation can be written as

$Y=\left\{\begin{array}{l}0 \text { (repeated root) } \\ \pm c_{m 0}\end{array}\right.$

The corresponding solutions for the Eq. (35) are,

$\lambda=\left\{\begin{array}{l}u_{g} \quad \text { (repeated root) } \\ u_{g} \pm c_{m 0}\end{array}\right.$

The roots for the zero slip velocity are real and hence the system is hyperbolic for this case.

\subsection{Non-dimensional characteristic equation}

For the case, $\Delta u \neq 0$, one can define, $Z=\Delta u Y$, to rewrite Eq. (40) as follows, 


$$
-\frac{\Delta u^{4}}{c_{m 0}^{2}}\left[\left(1-Z^{2}\right)^{2}\right]+\frac{\Delta u^{2}}{\left(\alpha_{l} \rho_{g}+\alpha_{g} \rho_{l}\right)}\left[\alpha_{l} \rho_{g}(1-Z)^{2}+\alpha_{g} \rho_{l}(1+Z)^{2}\right]=0
$$

Dividing by $\Delta u^{2}$, the above equation becomes,

$$
-\frac{\Delta u^{2}}{c_{m 0}^{2}}\left[\left(1-Z^{2}\right)^{2}\right]+\frac{1}{\left(\alpha_{l} \rho_{g}+\alpha_{g} \rho_{l}\right)}\left[\alpha_{l} \rho_{g}(1-Z)^{2}+\alpha_{g} \rho_{l}(1+Z)^{2}\right]=0
$$

Rearranging the terms in the above equation, one gets,

$$
-\frac{\Delta u^{2}}{c_{m 0}^{2}}\left[\left(1-Z^{2}\right)^{2}\right]+\left[Z^{2}-2 \frac{\alpha_{l} \rho_{g}-\alpha_{g} \rho_{l}}{\left(\alpha_{l} \rho_{g}+\alpha_{g} \rho_{l}\right)} Z+1\right]=0
$$

Defining the slip Mach number, $M_{s}$, as,

$$
\frac{\Delta u^{2}}{c_{m 0}^{2}}=M_{s}^{2}
$$

and

$$
\begin{aligned}
& r=\frac{\alpha_{l} \rho_{g}-\alpha_{g} \rho_{l}}{\alpha_{l} \rho_{g}+\alpha_{g} \rho_{l}} \\
& M_{s}^{2}\left[-\left(1-Z^{2}\right)^{2}\right]+\left[Z^{2}-2 r Z+1\right]=0
\end{aligned}
$$

It should be noted that $r^{2} \leq 1$. Also, $r^{2}=1$ when either $\alpha_{g}$ or $\alpha_{l}$ is zero.

From the Eq. (49) it is clear that for the small slip velocity i.e. $M_{s}^{2}<<1$, the effect of the compressibility on the hyperbolicity of the system can be ignored. It needs to be emphasized that $c_{g}^{2} \leq c_{m}^{2} \leq c_{l}^{2}$. Therefore, in the applications in which $\Delta u^{2}<<c_{g}^{2}$, the abovementioned compressibility effect can be ignored irrespective of the void fraction. In most applications, including the nuclear reactors $\Delta u^{2}$ is much smaller than $c_{g}^{2}$. Hence, the compressibility effect on the hyperbolicity can be ignored even if the compressible equations need to be solved for the flow simulations.

\subsection{Numerical Analysis of the Compressibility Effect}

In order to verify the above discussion, the minimum value of the $p_{i}$ required for the compressible two-phase two-fluid model to be hyperbolic is numerically calculated. These calculations are carried out for the steam water mixture assumed to be at thermal 
equilibrium (i.e. the values are taken along the saturation curve). The required $p_{i}$ for the corresponding incompressible flow is calculated using Eq. (33). The Table 1 gives the values of $\sigma$ which are the ratios of the $p_{i}$ obtained for the compressible flow to those obtained for the incompressible flows. It can be seen from the Table that for the small values of $M_{s}$, the value of $\sigma$ is close to 1 showing the compressibility has negligible effect on the hyperbolicity of the system when slip Mach number is small.

\begin{tabular}{|c|c|c|c|c|c|c|c|}
\hline $\begin{array}{c}\left|u_{g}-u_{l}\right| \rightarrow \\
\alpha_{g} \downarrow\end{array}$ & $10 \mathrm{~m} / \mathrm{s}$ & $20 \mathrm{~m} / \mathrm{s}$ & $30 \mathrm{~m} / \mathrm{s}$ & $40 \mathrm{~m} / \mathrm{s}$ & $60 \mathrm{~m} / \mathrm{s}$ & $80 \mathrm{~m} / \mathrm{s}$ & $100 \mathrm{~m} / \mathrm{s}$ \\
\hline 0.1 & 1.000 & 1.000 & 1.000 & 1.001 & 1.002 & 1.003 & 1.004 \\
\hline 0.5 & 0.011 & 0.021 & 0.031 & 0.043 & 0.063 & 0.084 & 0.105 \\
\hline 0.9 & 1.000 & 1.001 & 1.002 & 1.004 & 1.008 & 1.015 & 1.023 \\
& 0.011 & 0.021 & 0.032 & 0.043 & 0.063 & 0.084 & 0.105 \\
\hline \multirow{2}{*}{0.000} & 1.002 & 1.004 & 1.007 & 1.015 & 1.027 & 1.042 \\
& 0.011 & 0.021 & 0.032 & 0.043 & 0.063 & 0.084 & 0.105 \\
\hline
\end{tabular}

(a)

\begin{tabular}{|c|c|c|c|c|c|c|c|}
\hline $\begin{array}{c}\left|u_{g}-u_{l}\right| \rightarrow \\
\alpha_{g} \downarrow\end{array}$ & $10 \mathrm{~m} / \mathrm{s}$ & $20 \mathrm{~m} / \mathrm{s}$ & $30 \mathrm{~m} / \mathrm{s}$ & $40 \mathrm{~m} / \mathrm{s}$ & $60 \mathrm{~m} / \mathrm{s}$ & $80 \mathrm{~m} / \mathrm{s}$ & $100 \mathrm{~m} / \mathrm{s}$ \\
\hline 0.1 & 1.000 & 0.9998 & 0.9996 & 0.9993 & 0.9985 & 0.9973 & 0.9959 \\
& 0.009 & 0.018 & 0.027 & 0.036 & 0.054 & 0.071 & 0.089 \\
\hline \multirow{2}{*}{0.5} & 1.000 & 1.001 & 1.002 & 1.003 & 1.006 & 1.011 & 1.017 \\
& 0.010 & 0.020 & 0.030 & 0.040 & 0.061 & 0.081 & 0.101 \\
\hline \multirow{2}{*}{0.9} & 1.000 & 1.001 & 1.003 & 1.006 & 1.014 & 1.024 & 1.039 \\
& 0.011 & 0.020 & 0.031 & 0.042 & 0.063 & 0.082 & 0.103 \\
\hline
\end{tabular}

(b)

\begin{tabular}{|c|c|c|c|c|c|c|c|}
\hline $\begin{array}{c}\left|u_{g}-u_{l}\right| \rightarrow \\
\alpha_{g} \downarrow\end{array}$ & $10 \mathrm{~m} / \mathrm{s}$ & $20 \mathrm{~m} / \mathrm{s}$ & $30 \mathrm{~m} / \mathrm{s}$ & $40 \mathrm{~m} / \mathrm{s}$ & $60 \mathrm{~m} / \mathrm{s}$ & $80 \mathrm{~m} / \mathrm{s}$ & $100 \mathrm{~m} / \mathrm{s}$ \\
\hline 0.1 & 1.000 & 1.000 & 1.000 & 0.9999 & 0.9999 & 0.9999 & 0.9998 \\
& 0.009 & 0.019 & 0.028 & 0.038 & 0.056 & 0.075 & 0.091 \\
\hline 0.5 & 1.000 & 1.001 & 1.001 & 1.002 & 1.005 & 1.009 & 1.014 \\
& 0.011 & 0.022 & 0.033 & 0.044 & 0.066 & 0.089 & 0.110 \\
\hline \multirow{2}{*}{0.9} & 1.000 & 1.002 & 1.004 & 1.007 & 1.016 & 1.029 & 1.046 \\
& 0.012 & 0.022 & 0.034 & 0.046 & 0.068 & 0.091 & 0.120 \\
\hline
\end{tabular}

(c)

Table 1. Values of $\sigma$ (upper values) and $M_{s}^{2}$ (lower values) at the pressure of (a) 0.1 MPa (Atm. Pressure); (b) 7.5 MPa (BWR Pressure); (c) $15 \mathrm{MPa}$ (PWR Pressure)

It should be noted that for the reactor applications the slip velocity even with a conservative estimate is less than $20 \mathrm{~m} / \mathrm{s}$. Therefore, from the Table 1 it can be concluded 
that the compressibility effect on the hyperbolicity can be safely ignored for the reactor applications.

\section{Numerical Solutions of the Benchmark problem}

\subsection{Water Faucet Problem}

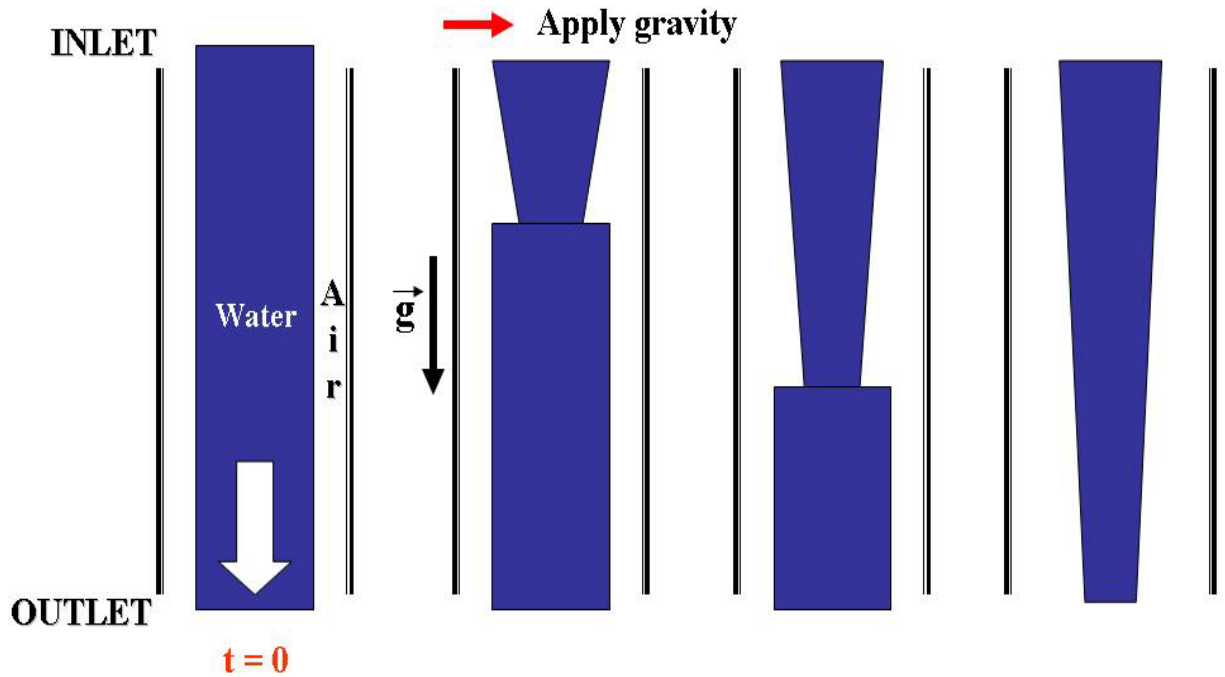

Fig. 1. Schematic of the water faucet problem

The water Faucet problem devised by Ransom [2] is used to study the analysis carried out in the previous sections. The schematic of the problem is shown in Fig. 1. The problem consists of a water jet in a cylindrical channel accelerating under the influence of gravity. Initially, the void fraction, the liquid velocity, the gas velocity and the pressure are considered to be uniform in the problem domain. The inlet values of void fraction, liquid velocity, gas velocity and the outlet pressure are constant for the transient.

\subsection{Numerical Results}

Numerical results are obtained for the inlet (and the initial) velocity and the void fraction of $5 \mathrm{~m} / \mathrm{s}$ and 0.2 , respectively. The length of the channel is $12 \mathrm{~m}$. The results are obtained for the outlet pressures of $7.5 \mathrm{Mpa}$ and $15 \mathrm{Mpa}$. The traditional numerical scheme for the reactor safety (used in the present study) is first order, both in space and time [1].

The results of the numerical simulations are given in Fig. 2. The results obtained with the lower values of $\sigma$ show large oscillations except in the case of the simulations carried out at the atmospheric pressure. The lack of the oscillations for the results with $\sigma=1$ confirm that this value of the $\sigma$ is sufficient for the making the model hyperbolic. It is also seen that $\sigma=0.9$ also give quite good results, especially at the lower pressure. However, it 
should be noted that if the numerical diffusion is decreased, the value of $\sigma=1$ will be needed.

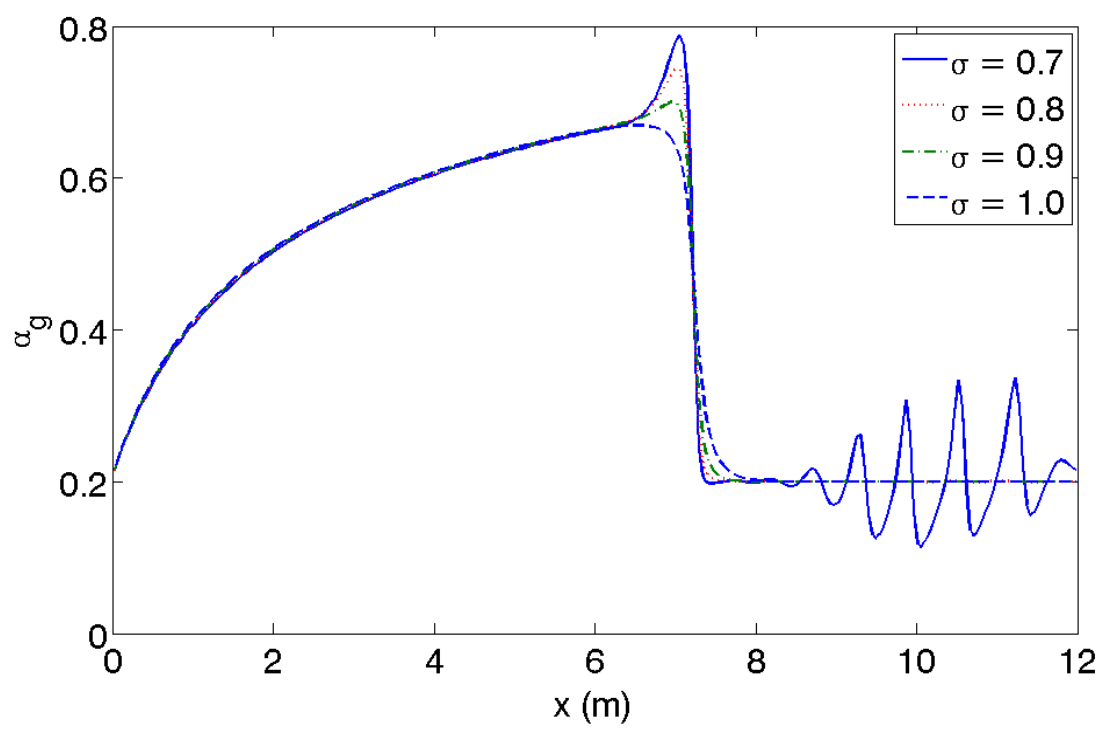

(a)

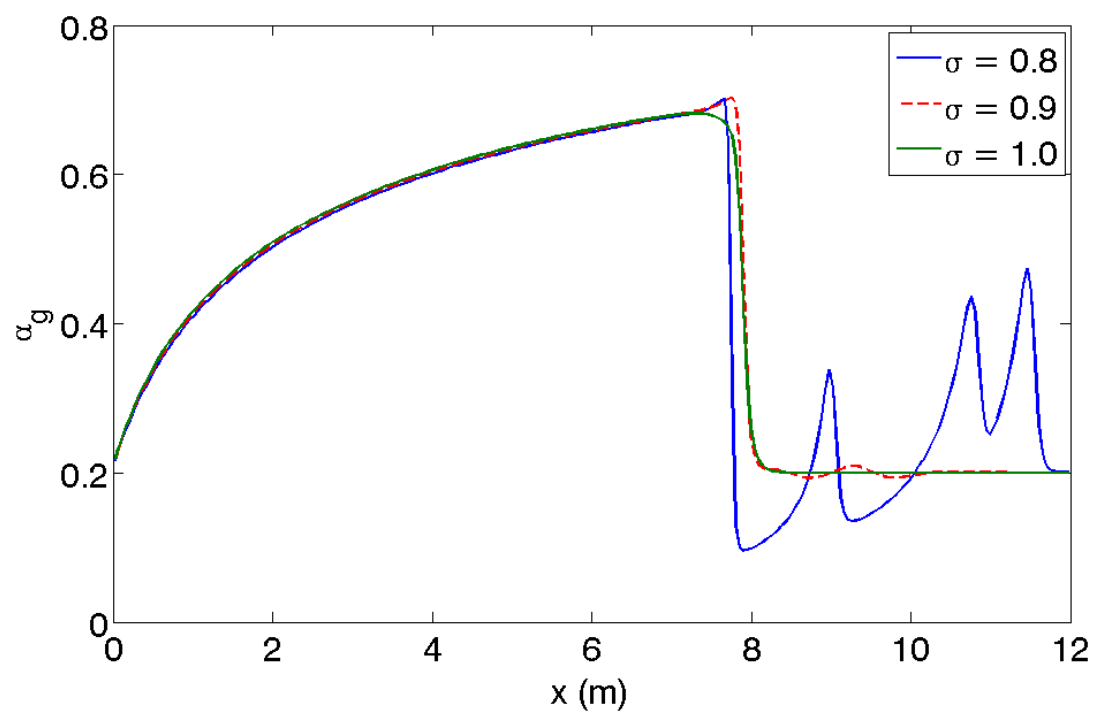

(b)

Fig. 2. The void fraction distribution for the water faucet problem with the inlet pressure of (a) 7.5 MPa (BWR Pressure);(b) $15 \mathrm{MPa}$ (PWR Pressure).

7. Series Solution of the characteristic equation for the small slip velocities

An analytical solution of the characteristic equation, which is a quartic polynomial 
equation, can be obtained. However, this solution is in the radical form and not very useful for any physical interpretation. As discussed earlier, for most of the applications value of $M_{s}$ is small. The roots of the characteristic equation can be found as a power series expansion about the point $M_{s}=0$. These roots are useful for obtaining choke flow criterion.

For $M_{s}=0$, the two roots of the characteristic equation, Eq. (49), are as follows,

$$
Z=r \pm \sqrt{r^{2}-1}
$$

These two roots are complex, except for $r^{2}=1$. (Note that $r^{2} \leq 1$ as discussed before). Since these roots are obtained by neglecting the compressible part of the equation these are same as those obtained for the incompressible flow. The corresponding values of the $\lambda$ (obtained using Eqs. (36), (38) and (48)) are same as that for the incompressible flow given in the Eq. (22).

The numerical solution can be obtained for Eq. (49) for given values of $M_{s}$ and $r$. From the roots obtained (using Mathematica ${ }^{\circledR}$ ) for small values of $M_{s}$, it is observed, that two of the roots approach the values given by Eq. (50) as $M_{s} \rightarrow 0$. The remaining two roots are real and approach the following values,

$Z=-r \pm \frac{1}{M_{s}}$

The corresponding values of the $\lambda$ can be obtained as (from Eqs. (36), (38) and (48)),

$$
\lambda=\frac{\left(u_{g} \alpha_{g} \rho_{l}+u_{l} \alpha_{l} \rho_{g}\right)}{\left(\alpha_{l} \rho_{g}+\alpha_{g} \rho_{l}\right)} \pm c_{m 0}
$$

Defining,

$$
u_{m 0}=\frac{\left(u_{g} \alpha_{g} \rho_{l}+u_{l} \alpha_{l} \rho_{g}\right)}{\left(\alpha_{l} \rho_{g}+\alpha_{g} \rho_{l}\right)}
$$

the eigenvalues can be written as,

$$
\lambda=u_{m 0} \pm c_{m 0}
$$

in a form similar to single phase eigenvalues.

It is interesting to note that, for $\alpha_{g} \rightarrow 0$ the above value reduces to $\lambda=u_{l} \pm c_{l}$ and for, 
$\alpha_{l} \rightarrow 0$ one can obtain that $\lambda=u_{g} \pm c_{g}$. These values are the eigenvalues for the single phase flows. Moreover, for $u_{g} \rightarrow u_{l}$, the above roots reduce to the roots for the zero slip velocity case [Eq.(42)]. It is also important to note that the four eigenvalues obtained by Trapp and Ransom (1982) [7] are same as those given in Eqs. (22) and (52) if virtual mass and thermal non-equilibrium terms are ignored.

The difference between the roots obtained numerically and those obtained from Eq. (51) is plotted in Fig. 3. From the plots it can be seen that error is $O\left(M_{s}\right)$. However, the residual $R$ of the non-dimensional characteristic equation, defined as follows

$$
R(Z) \equiv M_{s}^{2}\left[-\left(1-Z^{2}\right)^{2}\right]+\left[Z^{2}-2 r Z+1\right]
$$

has the following value

$$
R\left(-r \pm \frac{1}{M_{s}}\right)=\left(r^{2}-1\right)\left(-3 \pm 4 M_{s} r+\left(1-r^{2}\right) M_{s}^{2}\right)
$$

which does not go to zero as $M_{s} \rightarrow 0$.

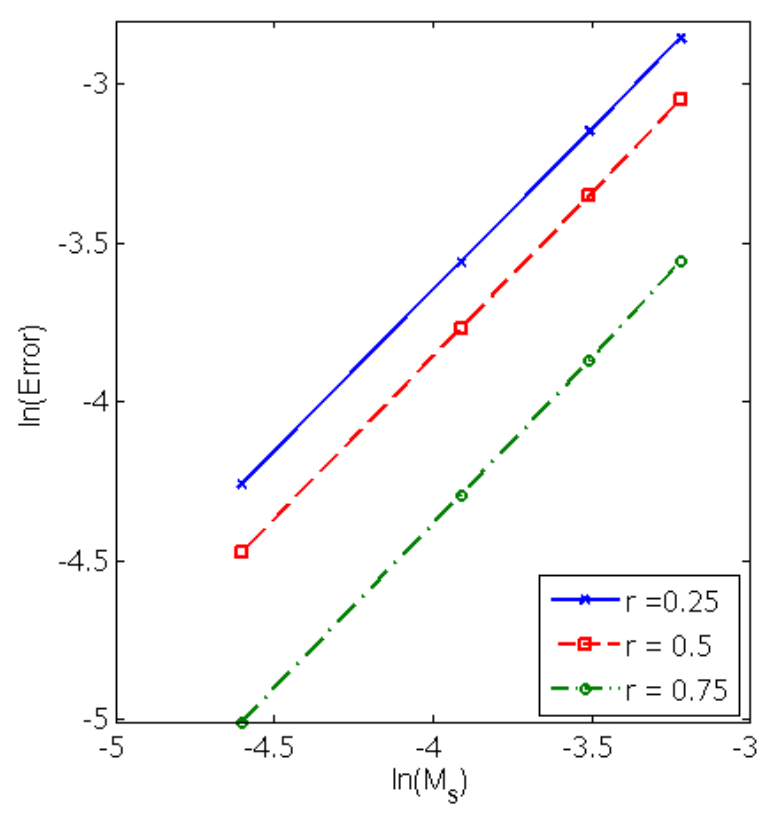

Fig. 3. The plot of error in the eigenvalue given by Eq. (51) as a function of $M_{s}$. The plot shows that error is $O\left(M_{s}\right)$.

In order to find higher order terms in the root of the characteristic equation, one can 
substitute $Z=-r \pm \frac{1}{M_{s}}+f_{1}(r) M_{s}$ in the non-dimensional characteristic equation to yield,

$$
R\left(-r \pm \frac{1}{M_{s}}+f_{1}(r) M_{s}\right)=3\left(1-r^{2}\right) \mp 2 f_{1}(r)+M_{s} r\left(\mp 4\left(1-r^{2}\right)+8 f_{1}(r)\right)+O\left(M_{s}^{2}\right)
$$

From the above equation it can be seen that choosing,

$$
f_{1}(r)= \pm \frac{3}{2}\left(1-r^{2}\right)
$$

will make the residual to be $O\left(M_{s}\right)$. The difference in the root obtained with

$Z=-r \pm \frac{1}{M_{s}} \pm \frac{3}{2}\left(1-r^{2}\right) M_{s}$

and the numerical solution is plotted in the Fig. 4. It can be seen that the error is $O\left(M_{s}^{2}\right)$. It is emphasized here that for the approximate root given in the Eq. (59) the error is $O\left(M_{s}^{2}\right)$ while the residual is $O\left(M_{s}\right)$. The behavior is similar to the root given by Eq. (51) in which case root has the error $O\left(M_{s}\right)$ while the residual is $O(1)$ [Eq. (56)]. Therefore, it is seen that the error in the root is one order lower than the residual.

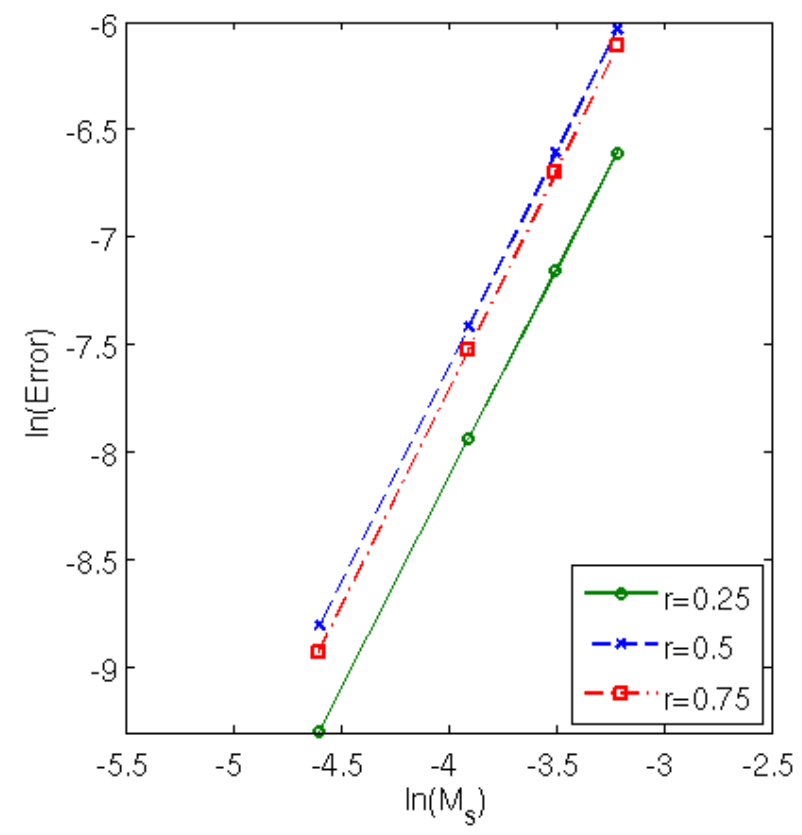

Fig. 4. The plot of error in the eigenvalue given by Eq. (59) as a function of $M_{s}$. The plot shows that error is $O\left(M_{s}\right)$. 
To obtain the next term in the series one can evaluate residual with

$$
Z=-r \pm \frac{1}{M_{s}} \pm \frac{3}{2}\left(1-r^{2}\right) M_{s}+f_{2}(r) M_{s}^{2}
$$

and then evaluate $f_{2}(r)$ such that $O\left(M_{s}\right)$ term in the residual is eliminated. In fact, the roots can be found with arbitrary accuracy (as long as $M_{s}<1$ ), by using the above process recursively. The first few terms are obtained for the two real roots are as follows,

$$
\begin{aligned}
Z= & -r \pm \frac{1}{M_{s}} \pm \frac{3}{2}\left(1-r^{2}\right) M_{s}+4 r\left(1-r^{2}\right) M_{s}^{2} \pm \frac{5}{8}\left(1-r^{2}\right)\left(21 r^{2}-5\right) M_{s}^{3} \\
& +24 r\left(1-r^{2}\right)\left(2 r^{2}-1\right) M_{s}^{4}+O\left(M_{s}^{5}\right)
\end{aligned}
$$

\subsection{Analysis of the real roots of the characteristic equation}

It can be seen that the third and fifth term in the series have the same sign as the $1 / M_{s}$ while the remaining terms do not change sign. Moreover, fourth and sixth term have $r$ as a factor. In the light of the above observation, the terms in the Eq. (61) are rearranged as follows,

$$
\begin{aligned}
Z=- & r\left\{1-4\left(1-r^{2}\right) M_{s}^{2}-24\left(1-r^{2}\right)\left(2 r^{2}-1\right) M_{s}^{4}+O\left(M_{s}^{6}\right)\right\} \\
& \pm \frac{1}{M_{s}}\left\{1+\frac{3}{2}\left(1-r^{2}\right) M_{s}^{2}+\frac{5}{8}\left(1-r^{2}\right)\left(21 r^{2}-5\right) M_{s}^{4}+O\left(M_{s}^{6}\right)\right\}
\end{aligned}
$$

In the above equation, terms in Eq. (61) are written as two different series. As $M_{s} \rightarrow 0$, the first series goes to $-r$ while the second series approaches $1 / M_{s}$.

Using equation (36) and (38), the values of the $\lambda$ are obtained corresponding to the $Z$ values in the Eq. (62) as follows,

$$
\begin{aligned}
\lambda= & u_{m 0}-r(\Delta u)\left\{-4\left(1-r^{2}\right) M_{s}^{2}-24\left(1-r^{2}\right)\left(2 r^{2}-1\right) M_{s}^{4}+O\left(M_{s}^{6}\right)\right\} \\
& \pm c_{m 0}\left\{1+\frac{3}{2}\left(1-r^{2}\right) M_{s}^{2}+\frac{5}{8}\left(1-r^{2}\right)\left(21 r^{2}-5\right) M_{s}^{4}+O\left(M_{s}^{6}\right)\right\}
\end{aligned}
$$

The definitions of the $u_{m 0}$ and $c_{m 0}$ used in the above equation are given in Eq. (53) and Eq. (47), respectively. The Eq. (63) can be written as,

$$
\lambda=u_{m} \pm c_{m}
$$


where,

$$
u_{m} \equiv u_{m 0}-r(\Delta u)\left\{-4\left(1-r^{2}\right) M_{s}^{2}-24\left(1-r^{2}\right)\left(2 r^{2}-1\right) M_{s}^{4}+O\left(M_{s}^{6}\right)\right\}
$$

and

$$
c_{m}=c_{m 0}\left\{1+\frac{3}{2}\left(1-r^{2}\right) M_{s}^{2}+\frac{5}{8}\left(1-r^{2}\right)\left(21 r^{2}-5\right) M_{s}^{4}+O\left(M_{s}^{6}\right)\right\}
$$

Note that as $\alpha_{g} \rightarrow 0, r^{2} \rightarrow 1, u_{m 0} \rightarrow u_{l}$ and $c_{m 0} \rightarrow c_{l}$. Therefore, the real eigenvalues approach $\lambda=u_{l} \pm c_{l}$. (Here, it is assumed that higher order terms also have a factor of $r^{2}-1$ in them). Similar result can be obtained for $\alpha_{l} \rightarrow 0$. Clearly, the roots approach the single phase eigenvalues in the abovementioned limits. Also, in case $u_{g}=u_{l}$, one gets, $u_{m 0}=u_{g}=u_{l}$ and $M_{s}=0$. Therefore, the two eigenvalues are given as, $\lambda=u_{g} \pm c_{m 0}$ same as those obtained earlier [Eq. (42)].

\subsection{Complex roots of the characteristic equation}

The two complex conjugate roots in the series form can be found in a similar manner as the real roots. The roots of the non-dimensional characteristic equation are as follows,

$$
\begin{aligned}
Z= & r\left\{1-4\left(1-r^{2}\right) M_{s}^{2}-24\left(1-r^{2}\right)\left(2 r^{2}-1\right) M_{s}^{4}+O\left(M_{s}^{6}\right)\right\} \\
& \pm \sqrt{r^{2}-1}\left\{1+\left(-2+4 r^{2}\right) M_{s}^{2}+6\left(1-8 r^{2}+r^{4}\right) M_{s}^{4}+O\left(M_{s}^{6}\right)\right\}
\end{aligned}
$$

It is interesting to note that, the series given in the first bracket in the equation is same as the series in the first bracket in the Eq. (62). The corresponding value of the $\lambda$ is as follows,

$$
\begin{aligned}
\lambda= & \frac{\left(u_{g} \alpha_{l} \rho_{g}+u_{l} \alpha_{g} \rho_{l}\right)}{\left(\alpha_{l} \rho_{g}+\alpha_{g} \rho_{l}\right)}+r \Delta u\left\{-4\left(1-r^{2}\right) M_{s}^{2}-24\left(1-r^{2}\right)\left(2 r^{2}-1\right) M_{s}^{4}+O\left(M_{s}^{6}\right)\right\} \\
& \pm \frac{\sqrt{-\left(u_{g}-u_{l}\right)^{2} \alpha_{g} \rho_{l} \alpha_{l} \rho_{g}}}{\left(\alpha_{l} \rho_{g}+\alpha_{g} \rho_{l}\right)}\left\{1+\left(-2+4 r^{2}\right) M_{s}^{2}+6\left(1-8 r^{2}+r^{4}\right) M_{s}^{4}+O\left(M_{s}^{6}\right)\right\}
\end{aligned}
$$

\subsection{Choke Flow Criterion for the two phase flows}

A choked condition exists when mass flow rate becomes independent of the downstream conditions. In other words, no information can propagate in the upstream direction under this condition. The real part of the eigenvalue represents velocity of the signal propagation and the imaginary part is the growth (or decay) rate of that signal. Therefore, if the real part of all the eigenvalues is positive then no signal propagates in the upstream 
direction (choosing downstream direction to be the positive direction). From the Eq. (64), such condition exists when $u_{m}=c_{m}$. Hence, this equation defines the choke flow criterion.

\section{Conclusions}

The effect of compressibility on the hyperbolicity of the two-phase two-fluid model is analyzed numerically as well as analytically. A non-dimensional characteristic equation is obtained for this model. The analysis of this equation shows that the compressibility effect on the hyperbolicity is negligible as long as the slip Mach number is small. This Mach number is shown to be small for the two-phase flows in the nuclear reactors and hence the compressibility effect on the hyperbolicity can be neglected for these reactors. Therefore, it can be safely assumed that the Stuhmiller model, which was developed to hyperbolize the incompressible two-phase two-fluid model, is sufficient for the low slip Mach number flow in the nuclear reactors. The numerical analysis of the water faucet problem shows that it is indeed the case.

Furthermore, the eigenvalues of the characteristics equation of the compressible twophase two-fluid model have been obtained as power expansion series. The choking condition exists when all the eigenvalues are positive. The abovementioned series solution is used to obtain a choking criterion for the two-phase flow.

\section{References}

1. U. S. N. R. C., RELAP 5/MOD 3.3 Code Manual Volume I, NUREG/CR-5535 Edition (December 2001).

2. V. H. RANSOM, V. MOUSSEAU, "Convergence and Accuracy Expectations for Two-phase Flow Simulations," Proc. of Canadian Nuclear Society Interanational Conference Simulation Methods in Nuclear Engineering, Montreal, Canada, April 18-20, (1990).

3. J. D. RAMSHAW, J. A. TRAPP, "Charesteristics, Stability, and ShortWavelength Phenomena in Two-Phase Flow Equation Systems," Nucl. Sci. Eng., 66, 93, (1978).

4. J. H. STUHMILLER, "The Influence of Interfacial Pressure on the Character of the Two-phase Flow Model Equations," Int. J. Multiphase Flow, 3, 551, (1977).

5. D. BESTION, "The Physical Closure Laws in the CATHARE Code," Nucl. Eng. Des., 124, 229, (1990). 
6. M.-S. LIOU, L. NGUYEN, C.-H. CHANG, S. SUSHCHIKH, R. R. NOURGALIEV, T. G. THEOFANOUS, "Hyperbolicity, Discontinuities, and Numerics of Two-Fluid Models", The 4th International Conference on Computational Fluid Dynamics, Ghent, Belgium, July 10-14, (2006).

7. J. A. TRAPP, V.H. RANSOM, "A Choked Flow Calculation Criterion for NonHomogeneous, Non-Equilibrium, Two-phase Flows", Int. J. Multiphase Flow, $\mathbf{8}(6), 669,(1982)$ 